\title{
5-DOF dynamic model of vehicle shimmy system with clearance at universal joint in steering handling mechanism
}

\author{
Jianwei Lu ${ }^{\mathrm{a}, \mathrm{b}, *}$, Yi Xu ${ }^{\mathrm{a}}$, Chen $\mathrm{Hu}^{\mathrm{a}}$, Alexander F. Vakakis ${ }^{\mathrm{b}}$ and Lawrence A. Bergman ${ }^{\mathrm{c}}$ \\ ${ }^{a}$ School of Mechanical and Automotive Engineering, Hefei University of Technology, Hefei, Anhui, China \\ ${ }^{\mathrm{b}}$ Department of Mechanical Science and Engineering, University of Illinois at Urbana-Champaign, Urbana, IL, \\ USA \\ ${ }^{\mathrm{c}}$ Department of Aerospace Engineering, University of Illinois at Urbana-Champaign, Urbana, IL, USA
}

Received 2 October 2012

Revised 11 January 2013

Accepted 13 April 2013

\begin{abstract}
A 5-DOF dynamic model of vehicle shimmy system with clearance in universal joint of steering handling mechanism is presented. The sub model of cross shaft universal joint with clearance is built based on Hertz' theory, and two-state model is applied to describe the contact force. The sub model of the universal joint is combined with the simplified dynamic model of steering system, and a 5-DOF dynamic model of vehicle shimmy system with consideration of assembling clearance in universal joint of steering handling mechanism is presented. Based on this model, numerical analysis is carried out to evaluate the influence of clearance in universal joint on the dynamic behavior of the vehicle shimmy system. The results show that the clearance and some other parameters, such as vehicle speed, have coupled contribution to the dynamic behavior of the vehicle shimmy system. The conclusions provide theoretical basis for effective attenuation of vehicle shimmy, especially for those in-service vehicles.
\end{abstract}

Keywords: Universal joint, clearance, nonlinear, vehicle shimmy

\section{Introduction}

Vehicle shimmy can be found widely in vehicles, especially in in-service vehicles due to wear of steering mechanism. Usually, slight vehicle shimmy does not lead to severe consequence, and it can be neglected. Severe vehicle shimmy is usually manifested as continuous shaking of steering wheel and continuous shimmy of the front wheels. Once it happens, the handling stability and NVH (Noise Vibration and Harshness) performance of the vehicle will deteriorates, and it will have great negative influence on the traffic safety. At the same time, severe vehicle shimmy usually leads to increase of dynamic load on the steering mechanism. As a result, wear at kinematic pair of steering mechanism increases, and the reliability of the mechanism decreases. In addition, severe shimmy of front wheels lead to increase of rolling resistance of the tires, which results in decreased power performance and fuel economy performance of the vehicle. As a result, further research on vehicle shimmy and its prevention has been paid much more attention in recent years with growing requirements on the performance of vehicle.

The early research on vehicle shimmy can be traced back to 1930's. In 1931, Bradley and Allen noticed the phenomenon. They found that it is related with the tire lateral characteristic, and explored the researches on vehicle shimmy [12]. In general, the modes of vehicle shimmy can be grouped into three categories:

\footnotetext{
*Corresponding author: Jianwei Lu, School of Mechanical and Automotive Engineering, Hefei University of Technology, Hefei 230009, Anhui, China. Tel.: +86 55162901339 8828; E-mail: jwlu75@yahoo.com.cn.
} 
(1) Vehicle shimmy due to mechanics of tire, which refers to vehicle shimmy dominantly due to the side slide character of pneumatic tire.

(2) Vehicle shimmy due to structural parameters, which refers to vehicle shimmy dominantly due to incompatible dynamical parameters of the steering mechanism and suspension.

(3) Vehicle shimmy due to clearance, which refers to vehicle shimmy dominantly due to kinematic pair clearance in steering mechanism.

Usually, all of the three modes may have contributions to final vehicle shimmy simultaneously. While in some cases, one of them may be dominant. In new vehicles, the first two modes are dominant, while the latter is more effective in in-service vehicles. Since defects of new vehicles are paid much more attention than those of in-service vehicles, most of the previous research on vehicle shimmy has been focused on the former two modes.

For example, Demic [1] analyzed the effect of the steering system parameters on the wheel shimmy of heavy duty truck based on the interaction between the steering system and suspension. Kovacs [2] presented the simulation of vehicle shimmy for a mid-size truck with focus on a computational analysis tool that measured the power and corresponding energy dissipation. Stepan [3] proved that the front wheel shimmy would be out of control by Hopf bifurcation with variation of the parameters of the system, and provided a means to control the unstable wheel shimming through designing a globally stabilizing controller with feedback linearization. Gordon [4] presented a perturbation analysis of nonlinear wheel shimmy in aircraft landing gear, which showed that stable or unstable limit cycles can exist for taxi speeds above or below a critical value with stability of the limit cycles being determined by the sign of a computed coefficient. Lin [5] presented a nonlinear shimmy model of three-DOF, and proved that steering shimmy is a vibration phenomenon of stable limit cycle occurring after Hopf bifurcation. Virgile [6] presented an analytical model for the steering wheel vibration, which was verified with road testing, and the shimmy was found to be related to the vehicle speed and tire imbalance. Zhou [7] developed incremental harmonic balance (IHB) formulations for a four-DOF aircraft wheel shimmy system with combined Coulomb and velocity-squared damping, which was also applicable for steering wheel shimmy system of vehicles. Che [8] provided a mathematical model for wheel shimmy of off-road vehicles. Based on this model, the structural parameters of the vehicle were optimized to eliminate the wheel shimmy. With further development of nonlinear dynamics, some other research on vehicle shimmy system has focused on the nonlinear factors in the system such as the nonlinear lateral characters of tire [9]. For example, Liu [10] presented a dynamic model of steering system by consideration of nonlinear lateral force of tire, and discussed the stability of the steering system. Li [11] discussed the bifurcation characters of the steering wheel with nonlinear dynamic tire model.

All these research mentioned above provided good basis for causation analysis of vehicle shimmy, and made contributions to successful fault diagnosis and attenuation of vehicle shimmy in some cases. While the clearance in kinematic pair of steering mechanism has not been paid attention in all these research.

According to previous research of the authors' group, it has been verified that there are some obvious differences in the dynamic behavior of the vehicle shimmy system with or without consideration of clearances in steering mechanism [12-14]. Cross shaft universal joint is widely used in steering handling mechanism, and assembling clearance is required to prevent movement interference. Therefore, in this paper, the influence of clearance in universal joint of steering handling mechanism on the dynamic behavior of vehicle shimmy system is discussed. The universal joint with clearance between the cross shaft neck and universal joint fork is modeled based on Hertz law, and a 5-DOF dynamic model of vehicle shimmy system with consideration of clearance in handling mechanism is presented. Numerical analysis of the dynamic response of the vehicle shimmy system with clearance in handling mechanism is carried out, and influence of the clearance on the dynamic behavior of the vehicle shimmy system is evaluated. This will provide theoretical basis for improvement of vehicle shimmy control.

\section{Dynamic model of universal joint with clearance}

The diagram of cross shaft type universal joint is shown in Fig. 1, in which, the angular displacement of driving joint fork is $\varphi_{1}$, the angular displacement of driven joint fork is $\phi$, and the angle between the driving and driven joint forks is $\beta$. Usually there is radial clearance between the cross shaft neck and the inner circle of the journal due to assembling tolerance. To simplify the model, it is assumed that there is radial clearance between the cross shaft neck 


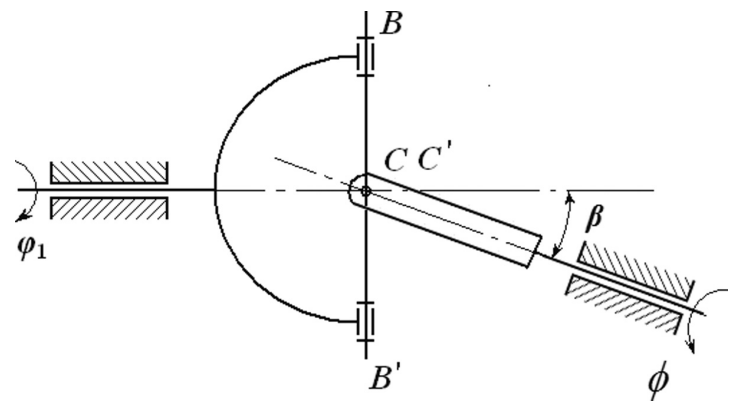

Fig. 1. Schematic of cross shaft universal joint.

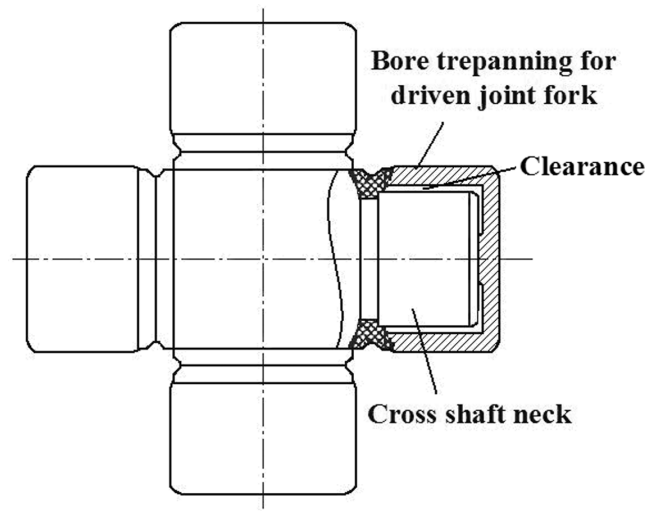

Fig. 2. Schematic of clearance at the cross shaft neck.

and the bore trepanning of the driving joint fork, as shown in Fig. 2, while it is assumed that there is no clearance in driven joint fork.

The constraint on the motion of cross shaft can be given as

$$
\tan \varphi_{s}=\tan \varphi_{r} \cos \beta
$$

In which, $\varphi_{s}$ is the angular displacement between the cross shaft and the connection neck of driving joint fork, $\varphi_{r}$ is the angular displacement between the cross shaft and the connection neck of driven joint fork. Since there is no clearance between the cross shaft neck and the driven joint fork, it can be deduced that $\phi=\varphi_{r}$. Therefore

$$
\tan \varphi_{s}=\tan \phi \cos \beta
$$

As a result, the relationship between $\dot{\varphi}_{s}$ and $\dot{\phi}$ can be given by

$$
\dot{\varphi}_{s}=\frac{\cos \beta}{\cos ^{2} \phi+\sin ^{2} \phi \cos ^{2} \beta} \dot{\phi}
$$

and the relationship between $\ddot{\varphi}_{s}$ and $\ddot{\phi}$ can be written as

$$
\ddot{\varphi}_{s}=\cos \beta \ddot{\phi}-2 \dot{\phi}^{2} \frac{\sin \phi \cos \phi \sin ^{2} \beta \cos \beta}{\left(\cos ^{2} \phi+\sin ^{2} \phi \cos ^{2} \beta\right)}
$$

The transmission error between the cross shaft and the driving joint fork is given by

$$
\Delta \varphi=\varphi_{1}-\varphi_{s}
$$

The contact force between the cross shaft and the driving joint fork is defined based on the two-state model. The relative distance between the cross shaft neck and bore trepanning of the driving joint fork can be written as

$$
\Delta r=\Delta \varphi R_{s}
$$

In which, $R_{s}$ is the distance from the center of the cross shaft to the end of the shaft. There is needle bearing between the driving joint fork and the cross shaft neck, and the interaction force between them can be described with an equivalent massless spring-damped system, which is given by

$$
F_{d}=F_{k}+F_{c}
$$

in which, the elastic force is given by

$$
F_{k}(\Delta r)=k f_{k}(\Delta r)
$$

where, $k$ is the equivalent contact stiffness, which can be written as

$$
k=\frac{\pi b}{2\left(g_{a}+g_{b}\right)}
$$

The function $f_{k}(\Delta r)$ is given by [12-14] 


$$
f_{k}(\Delta r)= \begin{cases}\Delta r-r & \Delta r>r \\ 0 & -r<\Delta r<r \\ \Delta r+r & \Delta r<-r\end{cases}
$$

where, $r$ is the clearance at the end of the cross shaft, $g_{a}=\frac{1-\mu_{1}^{2}}{E_{1}}, g_{b}=\frac{1-\mu_{2}^{2}}{E_{2}} \cdot \mu_{1}, \mu_{2}$ are Poisson ratio of the materials of the two contact surfaces, and $E_{1}, E_{2}$ are their modulus of elasticity.

The equivalent damping force at the contact surface can be written as

$$
F_{c}(\Delta r)=c f_{c}(\Delta r)
$$

where, $c$ is the equivalent damping coefficient, and it is given by [12-14]

$$
f_{c}(\Delta r)= \begin{cases}\Delta \dot{r} & |\Delta r|>r \\ 0 & |\Delta r|<r\end{cases}
$$

The equivalent damping coefficient $c$ is given by [13]

$$
c=\frac{2 \sqrt{m k \ln H}}{\sqrt{(2 \ln H)^{2}-\pi}}
$$

where, $H$ is coefficient of restitution.

The equivalent mass $m$ is written as [13]

$$
m=\frac{I_{1} I_{2}^{\prime}}{R_{s}^{2}\left(I_{1}+I_{2}^{\prime}\right)}
$$

where, $I_{1}$ is moment of inertia of driving joint fork about its centerline; $I_{2}^{\prime}$ is the equivalent moment of inertia of the driven components about the centerline of the driving fork.

The dynamic model of cross shaft type universal joint can be established with lumped mass method based on the following assumptions.

(1) The stiffness of the bearings in the universal joint is high and thus the transverse oscillation can be neglected compared with the torsional oscillation of joint fork.

(2) Friction and viscous damping are neglected.

(3) The mass and moment of inertia of cross type shaft is much smaller than that of the joint forks, which can be neglected in the modeling.

As a result, the equation of motion of the driving joint fork can be written as

$$
I_{1} \ddot{\varphi}_{1}+T_{s}=T_{1}
$$

In which, $T_{1}$ is the driving moment applied on the driving joint fork; $T_{s}$ is the resistance moment, which is given by

$$
T_{s}=R_{s} c f_{c}(\Delta r)+R_{s} k f_{k}(\Delta r)
$$

In this research, we consider the driven joint fork to be free. Therefore, the rotational motion of the driven components is governed by

$$
I_{2} \ddot{\phi}=T_{2}
$$

In which, $I_{2}$ is moment of inertia of driven components about its centerline, and the relationship between $T_{s}$ and $T_{2}$ is

$$
T_{s} \dot{\varphi}_{s}=T_{2} \dot{\phi}
$$

Therefore, there is

$$
T_{2}=\frac{\cos \beta}{\cos ^{2} \phi+\sin ^{2} \phi \cos ^{2} \beta} T_{s}
$$




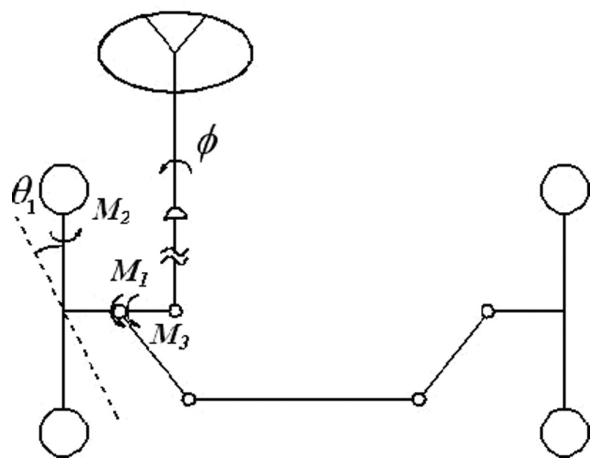

(a)

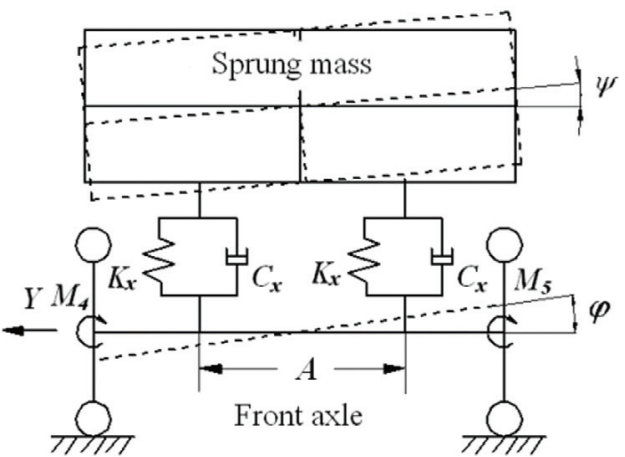

(b)

Fig. 3. Dynamic model of the vehicle shimmy system with clearance in universal joint.

\section{Dynamic model of vehicle shimmy system with clearance in handling mechanism}

The dynamic model of vehicle shimmy system with clearance in steering handling mechanism is shown in Fig. 3. In which, (a) is the structure of vehicle steering system, and (b) is the vehicle suspension. Where, $M_{1}$ is the moment applied on the left steering knuckle by handling mechanism; $M_{2}\left(M_{2}=F_{1} R \alpha\right)$ is the side moment of left front wheel applied on the king pin; $M_{3}\left(M_{3}=F_{1} n\right)$ is the aligning torque applied on left front wheel; $A$ is the distance between the front springs; $M_{4}$ is the side moment of left front wheel; $M_{5}$ is the side moment of right front wheel.

The dynamic model is a 5 DOF system: shimmy angle of left front wheel $\theta_{1}$, swing angle of front axle $\varphi$, rolling angle of sprung mass $\psi$, lateral displacement $Y$, and angular displacement of driven components of the universal joint $\phi$. The equations of motion are established with Lagrange equations based on the following assumptions.

(1) The steering wheel is free.

(2) There is no coupling effect between the vibration of rear wheel and that of front axle, which implies that it will make no influence on the shimmy of front wheels.

(3) The influence of caster angle $\alpha$ is considered, while other alignment parameters are neglected.

(4) The steering trapezium is in the horizontal plane, and the linkages of the steering mechanism are rigid links without elastic deformation.

(5) The moment of inertia of the steering tie rod, steering rocking arm and steering drag link are equalized to the moment of inertia of front wheels.

The kinetic energy of the system can be written as

$$
T=\frac{1}{2} I_{t}\left(\dot{\theta}_{1}^{2}+\dot{\theta}_{2}^{2}\right)+\frac{1}{2} I_{f} \dot{\varphi}^{2}+\frac{1}{2} I_{u} \dot{\psi}^{2}+\frac{1}{2} m_{w} \dot{Y}^{2}+\frac{1}{2} I_{1} \dot{\varphi}_{1}^{2}+\frac{1}{2} I_{2} \dot{\varphi}_{2}^{2}
$$

where, $I_{t}$ is equivalent moment of inertia of the front wheel about its king pin; $I_{f}$ is the moment of inertia of the front axle about its swing center; $I_{u}$ is the moment of inertia of the sprung mass about its inclination center; $m_{w}$ is the mass of the vehicle; $\theta_{2}$ is the shimmy angle of right front wheel. The relationship between $\theta_{1}$ and $\theta_{2}$ is determined by the geometric parameters of the steering trapezium.

The potential energy of the system is written as

$$
U=\frac{1}{2} K_{t v}\left(L \theta_{1} \alpha+\varphi \frac{B}{2}\right)^{2}+\frac{1}{2} K_{t v}\left(L \theta_{2} \alpha+\varphi \frac{B}{2}\right)^{2}+4\left(\frac{1}{2} K_{s} Y^{2}\right)+\frac{1}{2} K_{v} \theta_{1}^{2}+2\left\{\frac{1}{2} K_{x}\left[(\varphi-\psi) \frac{A}{2}\right]^{2}\right\}
$$

where, $K_{t v}$ is the vertical stiffness of the tire, $L$ is the distance from the interaction point of extension line of the kin pin and the ground to the symmetry plane; $B$ is the tread of front wheels; $K_{s}$ is the lateral stiffness of the tire; $K_{v}$ is the equivalent stiffness of the steering linkage about kin pin; $K_{x}$ is the stiffness of the suspension. 
The dissipation energy of the system is given by

$$
D=\frac{1}{2} C_{t}\left(\dot{\theta}_{1}+\dot{\varphi} \alpha\right)^{2}+\frac{1}{2} C_{t}\left(\dot{\theta}_{2}+\dot{\varphi} \alpha\right)^{2}+\frac{1}{2} C_{v} \dot{\theta}_{1}^{2}+2\left\{\frac{1}{2} C_{x}\left[(\dot{\varphi}-\dot{\psi}) \frac{A}{2}\right]^{2}\right\}
$$

In which, $C_{t}$ is the equivalent damping coefficient of the front wheel about kin pin; $C_{v}$ is the equivalent damping coefficient of the steering box about kin pin; $C_{x}$ is the damping coefficient of the suspension.

The general force corresponding to the shimmy of left front wheel is given by

$$
Q_{1}=-m_{t} \alpha \ddot{\varphi} \frac{B}{2} L-I_{x} \ddot{\varphi} \alpha+I_{i} \dot{\varphi} \frac{V}{R}+F_{1}(R \alpha+n)+M_{1}
$$

In which, $m_{t}$ is the mass of the tire, $I_{x}$ is moment of inertia of the front wheel about its vertical centerline through the mass center; $I_{i}$ is moment of inertia of the front wheel about its rotational shaft; $V$ is the vehicle speed; $R$ is the radius of the wheel; $F_{1}$ is the side force of left front wheel; $n$ is the tire drag; $M_{1}$ is the moment applied on the left steering knuckle by handling mechanism, and which can be written as

$$
M_{1}=T_{s} i_{w 1}=\left(R_{s} c f_{c}(\Delta r)+R_{s} k f_{k}(\Delta r)\right) i_{w 1}
$$

In which, $i_{w 1}$ is the equivalent transmission ratio of torque from driving joint fork of the universal joint to left steering wheel.

The general force corresponding to the swing angle of front axle is given by

$$
Q_{2}=-m_{t}\left(\ddot{\theta}_{1}+\ddot{\theta}_{2}\right) \frac{B}{2} L \alpha-I_{x}\left(\ddot{\theta}_{1}+\ddot{\theta}_{2}\right) \alpha-I_{i}\left(\dot{\theta}_{1}+\dot{\theta}_{2}\right) \frac{V}{R}-\left(F_{1}+F_{2}\right) R
$$

In which, $F_{2}$ is the side force of right front wheel.

The general force corresponding to the rolling angle of sprung mass is given by

$$
Q_{3}=0
$$

The general force corresponding to the lateral displacement of vehicle is given by

$$
Q_{4}=2\left(F_{1}+F_{2}\right)
$$

The general force corresponding to the angular displacement of driven components of universal joint is given by

$$
Q_{5}=T_{2}
$$

Based on the discussion presented above, the equations of motion of the system can be derived as the follows.

$$
\left\{\begin{array}{l}
I_{t} \ddot{\theta}_{1}+\left(C_{t}+C_{v}\right) \dot{\theta}_{1}+\left(K_{t v} L^{2} \alpha^{2}+K_{v}\right) \theta_{1}+\left(m_{t} L \alpha \frac{B}{2}+I_{x} \alpha\right) \ddot{\varphi}+\left(C_{t} \alpha-I_{i} \frac{V}{R}\right) \dot{\varphi} \\
\quad+K_{t v} L \alpha \frac{B}{2} \phi-F_{1}(R \alpha+n)-M_{1}=0 \\
I_{f} \ddot{\varphi}+\left(2 C_{t} \alpha^{2}+C_{x} \frac{A^{2}}{2}\right) \dot{\varphi}+\left(K_{t v} \frac{B^{2}}{2}+K_{x} \frac{A^{2}}{2}\right) \varphi+\left(m_{t} L \alpha \frac{B}{2}+I_{x} \alpha\right)\left(\ddot{\theta}_{1}+\ddot{\theta}_{2}\right) \\
\quad+\left(I_{i} \frac{V}{R}+C_{t} \alpha\right)\left(\dot{\theta}_{1}+\dot{\theta}_{2}\right)+K_{t v} L \alpha \frac{B}{2}\left(\theta_{1}+\theta_{2}\right)-C_{x} \frac{A^{2}}{2} \dot{\psi}-K_{x} \frac{A^{2}}{2} \psi+\left(F_{1}+F_{2}\right) R=0 \\
I_{u} \ddot{\psi}+C_{x} \frac{A^{2}}{2} \dot{\psi}+K_{x} \frac{A^{2}}{2} \psi-C_{x} \frac{A^{2}}{2} \dot{\varphi}-K_{x} \frac{A^{2}}{2} \varphi=0 \\
m_{w} \ddot{Y}+4 K_{s} Y-2\left(F_{1}+F_{2}\right)=0 \\
I_{2} \ddot{\phi}=T_{2}
\end{array}\right.
$$

In which, the first equation is motion of left front wheel around the king pin, the second one is for the front axle rolling motion, the third one is heeling motion of the mechanism above the suspension, the fourth one is for full vehicle lateral motion and the last one is for cross shaft rotation of steering universal joint.

Based on the equations of motion presented above, the dynamic analysis diagram of vehicle shimmy system with clearance in steering handling mechanism is shown in Fig. 4. 
Table 1

Values of parameters for numerical analysis

\begin{tabular}{clcl}
\hline Para- meter & Value/unit & Para- meter & Value $/ \mathrm{unit}$ \\
\hline$I_{t}$ & $180 / \mathrm{kg} \cdot \mathrm{m}^{2}$ & $m_{w}$ & $1500 / \mathrm{kg}$ \\
$I_{x}$ & $0.365 / \mathrm{kg} \cdot \mathrm{m}^{2}$ & $m_{t}$ & $16 / \mathrm{kg}$ \\
$I_{f}$ & $6.5 / \mathrm{kg} \cdot \mathrm{m}^{2}$ & $K_{t v}$ & $4 \mathrm{e} 5 / \mathrm{kN} \cdot \mathrm{m}^{-1}$ \\
$I_{u}$ & $1.05 \mathrm{e} 3 / \mathrm{kg} \cdot \mathrm{m}^{2}$ & $K_{v}$ & $1.5 \mathrm{e} 4 / \mathrm{kN} \cdot \mathrm{m} \cdot \mathrm{rad}^{-1}$ \\
$I_{i}$ & $4.86 / \mathrm{kg} \cdot \mathrm{m}^{2}$ & $K_{x}$ & $1.9 \mathrm{e} 4 / \mathrm{kN} \cdot \mathrm{m}^{-1}$ \\
$I_{s}$ & $1.415 / \mathrm{kg} \cdot \mathrm{m}^{2}$ & $L$ & $0.075 / \mathrm{m}$ \\
$C_{t}$ & $100 / \mathrm{N} \cdot \mathrm{m} \cdot \mathrm{s} \cdot \mathrm{rad}^{-1}$ & $l$ & $1.3426 / \mathrm{m}$ \\
$C_{v}$ & $40 / \mathrm{N} \cdot \mathrm{m} \cdot \mathrm{s} \cdot \mathrm{rad}^{-1}$ & $R$ & $0.4 / \mathrm{m}$ \\
$C_{x}$ & $897 / \mathrm{N} \cdot \mathrm{s} \cdot \mathrm{m}^{-1}$ & $A$ & $1.279 / \mathrm{m}$ \\
$n$ & $0.00289 / \mathrm{m}$ & $B$ & $1.608 / \mathrm{m}$ \\
$i_{w 1}$ & 12 & $V$ & $40 / \mathrm{km} \cdot \mathrm{h}^{-1}$ \\
\hline
\end{tabular}

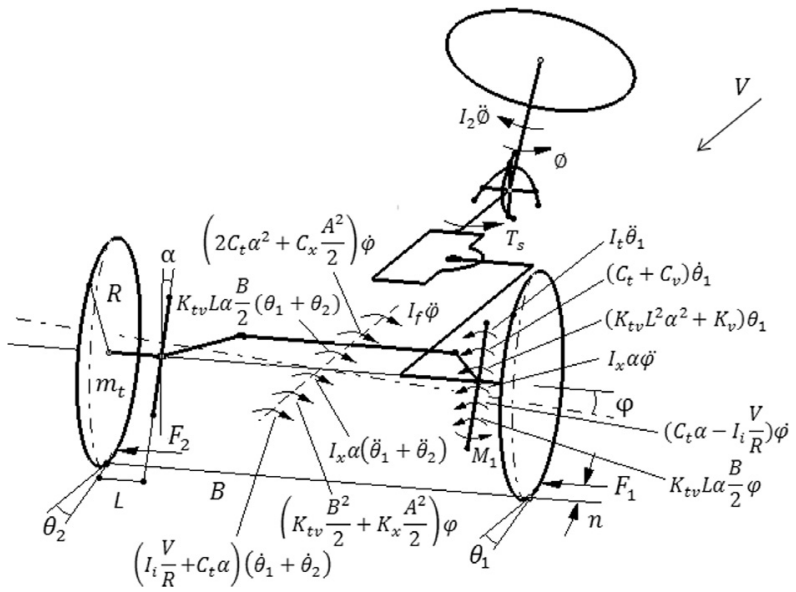

Fig. 4. Dynamic analysis diagram of the vehicle shimmy system with clearance in universal joint.
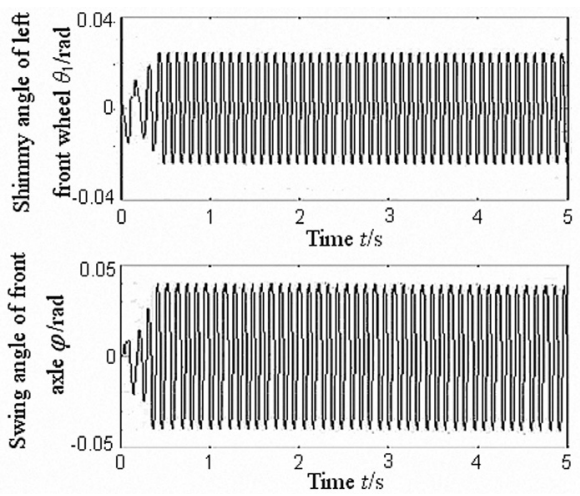

(a)
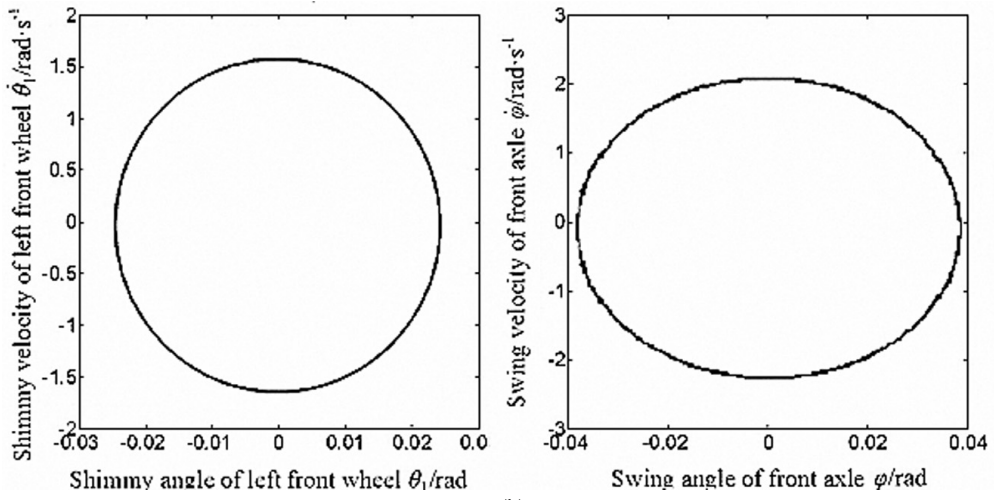

Fig. 5. Dynamic response of the shimmy system without clearance.
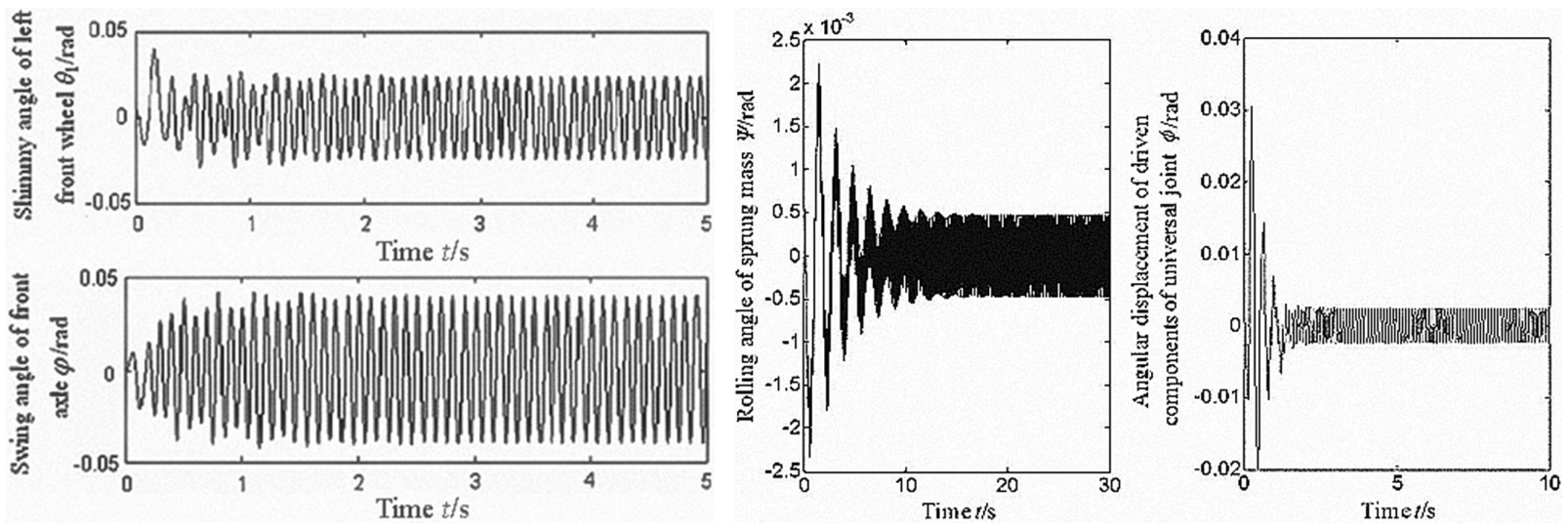

Fig. 6. Time history of the system response $(r=6 \mu \mathrm{m})$.

\section{Numerical analysis}

Based on the model presented above, numerical analysis is carried out to evaluate the influence of clearance on dynamic behavior of the vehicle shimmy system. In which, dynamic response of the system under an initial 

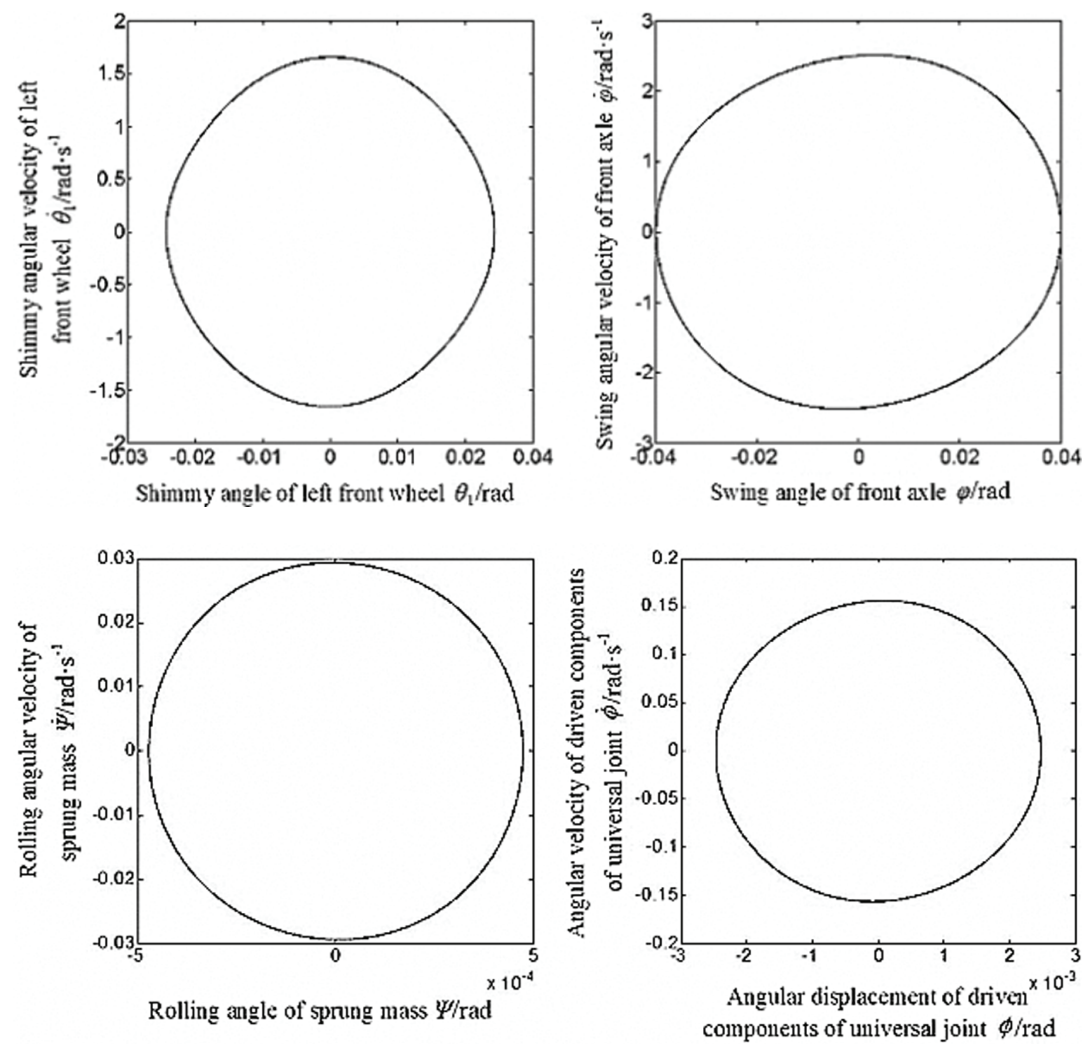

Fig. 7. Phase portrait of the system response $(r=6 \mu \mathrm{m})$.
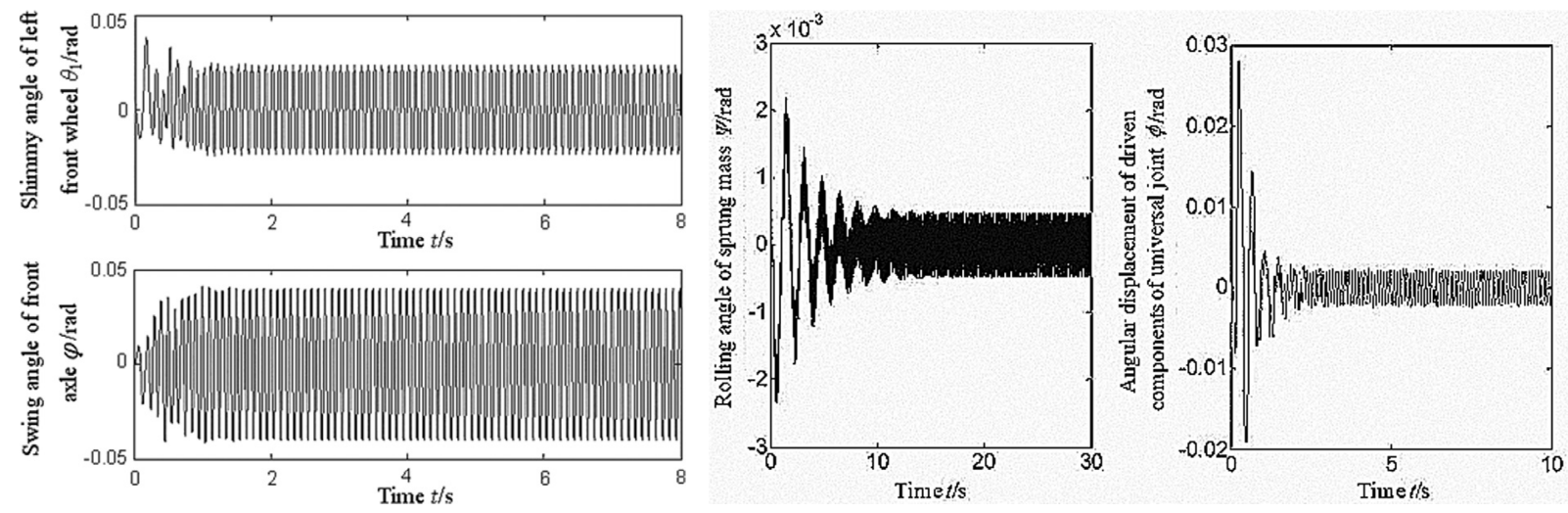

Fig. 8. Time history of the system response $(r=0.06 \mathrm{~mm})$.

displacement excitation on the left front wheel is evaluated with shimmy angle of left front wheel $\theta_{1}$, swing angle of front axle $\varphi$, rolling angle of sprung mass $\psi$, and angular displacement of driven components of the universal joint $\phi$. The initial angular displacement applied to left front wheel is $0.005 \mathrm{rad}$, and the numerical values of system parameters used for the numerical analysis are listed in Table 1.

The dynamic response of the system without clearance is shown in Fig. 5. In which, (a) is the time history of the system response and (b) the phase portrait. It can be seen that left front wheel and front axle step into stable limit cycle motion after a short time, i.e., system without clearance steps into stable limit cycle in a short time.

The time history of the system response with extremely small clearance $r=6 \mu \mathrm{m}$ is shown in Fig. 6 , and its phase 

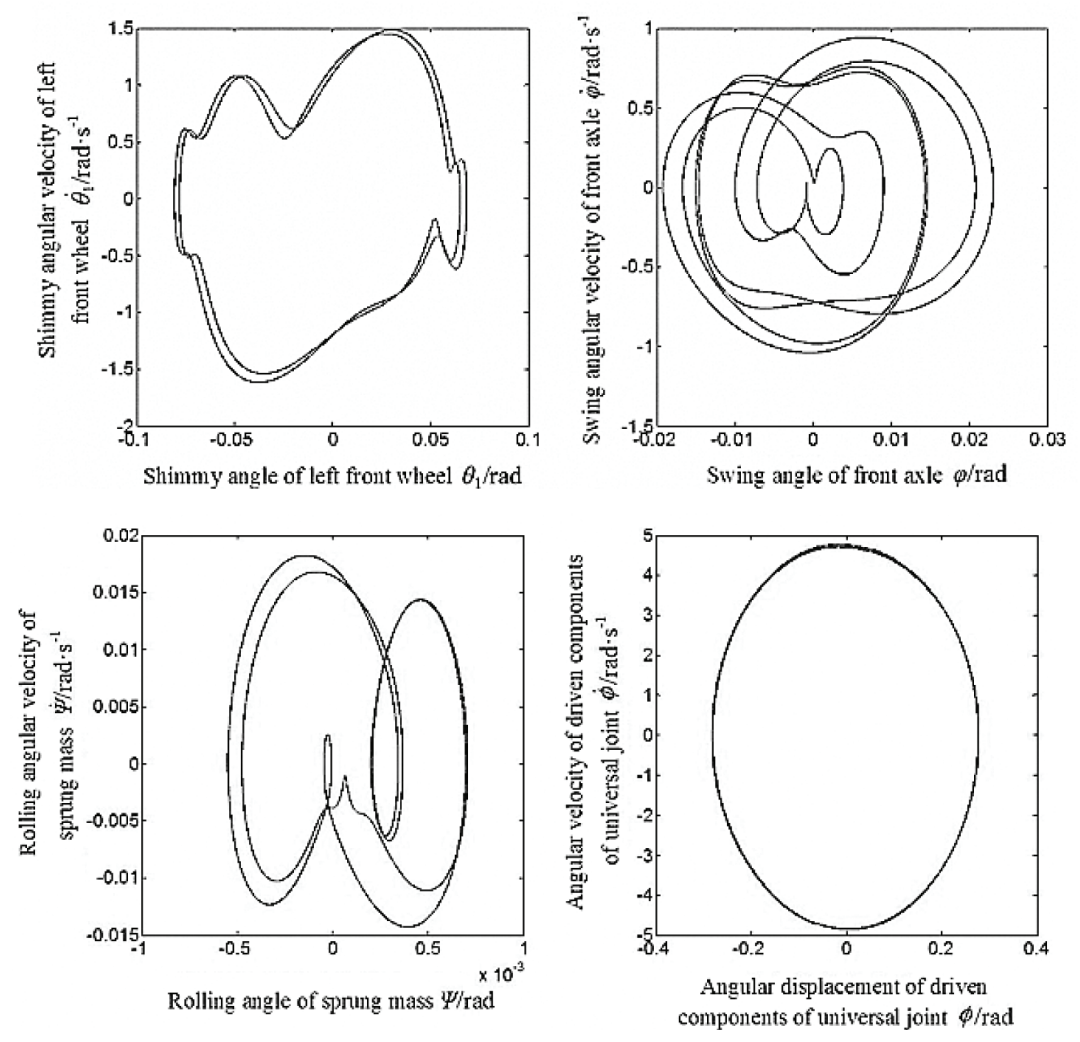

Fig. 9. Phase portrait of the system response $(r=0.06 \mathrm{~mm})$.
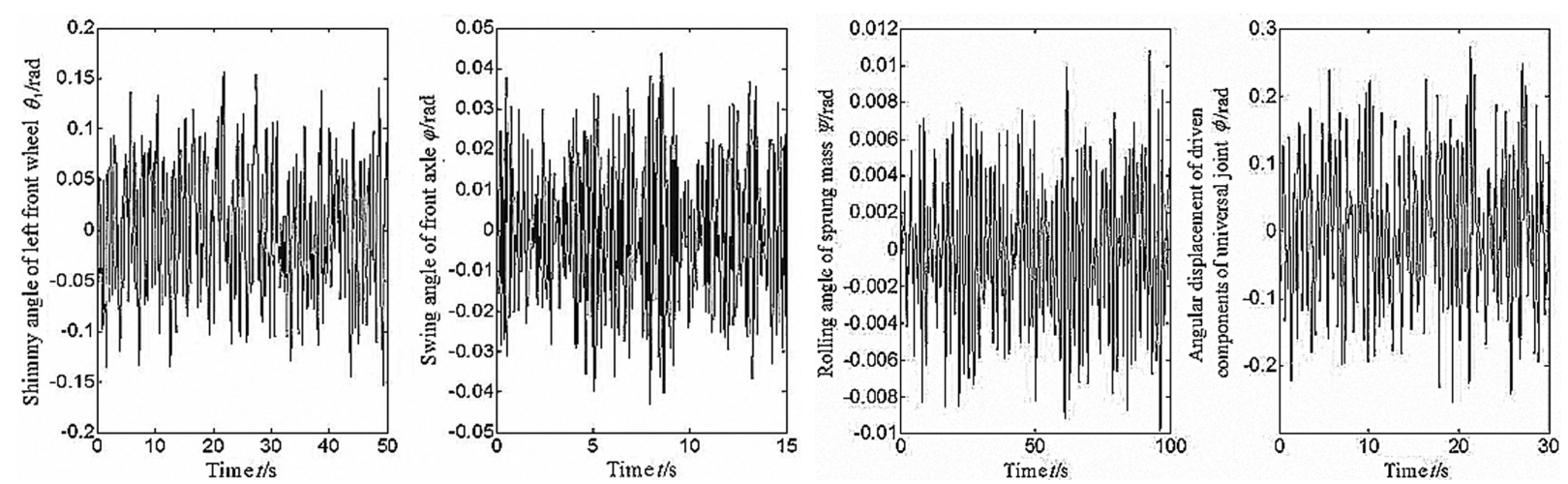

Fig. 10. Time history of the system response $(r=0.6 \mathrm{~mm})$.

portrait is shown in Fig. 7. It can be seen that left front wheel, front axle, and driven components of universal joint step into stable limit cycle motion after a short time, while it takes a longer time for sprung mass step into stable motion. The duration of transient state mainly depends on the parameters of the suspension, but the final motion of the sprung mass is a limit cycle.

The time history of the system response with small clearance $r=0.06 \mathrm{~mm}$ is shown in Fig. 8, and the phase portrait of the system response is shown in Fig. 9. Compared with the dynamic response of the system with extremely small clearance $r=6 \mu \mathrm{m}$, it can be seen that there is no significant difference in the displacement amplitude of the system, and neither in the transient duration. But the dynamic behavior of the system in stable motion begins to change. The dynamic response of the system is seen to be a multi period motion instead of single period limit cycle. 

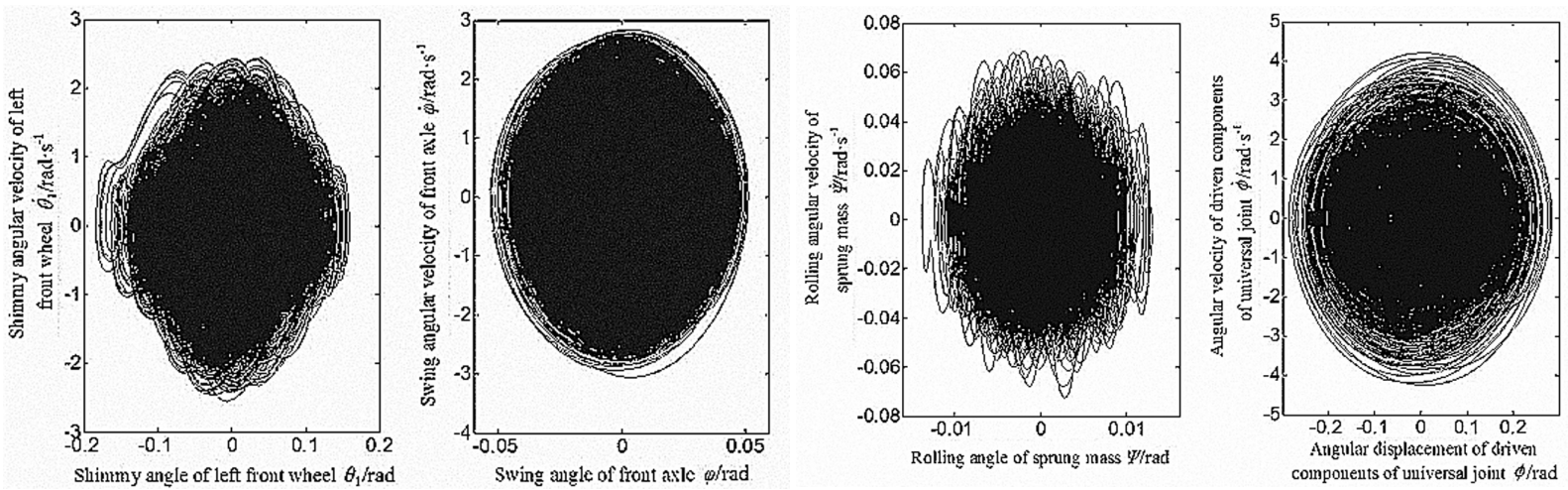

Fig. 11. Phase portrait of the system response $(r=0.6 \mathrm{~mm})$.

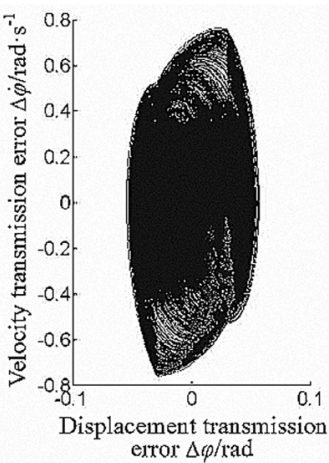

(a)

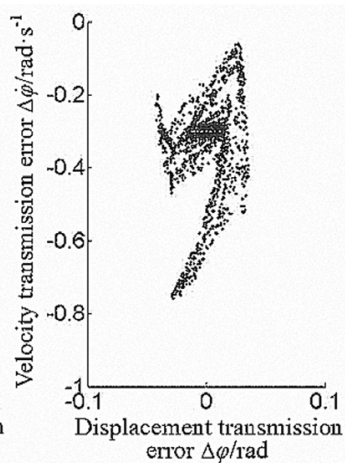

(b)

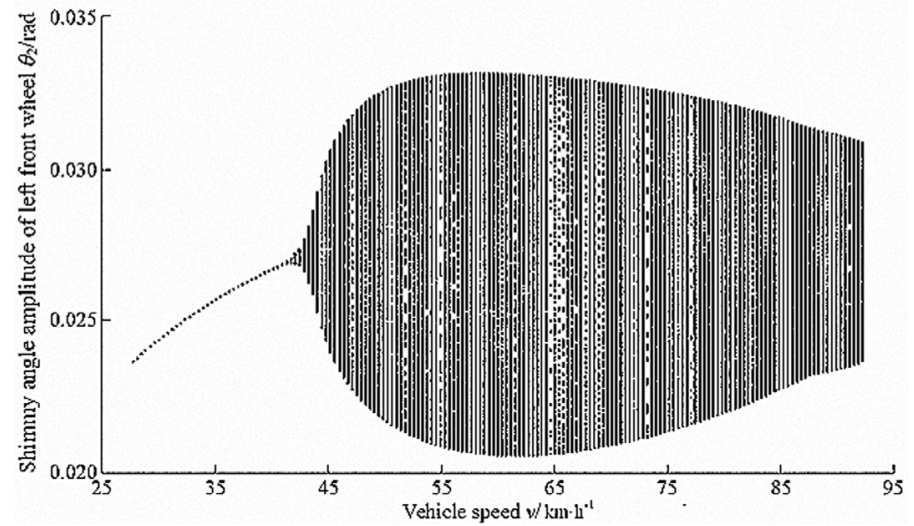

Fig. 13. Bifurcation diagram of the shimmy angle of left front wheel with variation of vehicle speed.

Fig. 12. Transmission character of the universal joint with clearance $(r=0.6 \mathrm{~mm})$.

If the clearance increases further to $r=0.6 \mathrm{~mm}$, the time history of the system response is shown in Fig. 10, and its phase portrait is shown in Fig. 11. It can be seen that the response of the shimmy system becomes instable and the displacement amplitude of the system response increases considerably. The phase trajectory of the system becomes disordered, which implies it steps into chaos. The transmission characteristic of the universal joint for this example is calculated as well, and the results are shown in Fig. 12. In which, (a) is phase portrait of the system, (b) is its Poincaré map. Since other parameters of the system are fixed, it can be deduced that the change in the dynamic behavior of the vehicle shimmy system is originated from the variation of transmission characteristic of the universal joint.

But it should be noted that the dynamic behavior of the shimmy system does not solely depend on the clearance of the universal joint. For the system with fixed clearance $r=0.06 \mathrm{~mm}$, the bifurcation diagram of the shimmy angle of left front wheel with variation of vehicle speed is shown in Fig. 13. It can be seen that the left front wheel is shown in periodic motion when the vehicle speed is below $43 \mathrm{~km} / \mathrm{h}$, but bifurcation occurs once the speed is higher than $43 \mathrm{~km} / \mathrm{h}$, and it begins to step into chaos.

In essence, it is verified that the clearance of the universal joint has great impact on the dynamic response of the shimmy system in addition to other system parameters, and the research on the coupling influence of the clearance and other parameters on the dynamic behavior of the shimmy system will be carried out in future.

\section{Conclusions}

A 5-DOF dynamic model of vehicle shimmy system with clearance in universal joint of steering handling mech- 
anism is presented. Based on this model, the influence of clearance on the dynamic behavior of the system is evaluated, and the following conclusions are derived.

(1) Compared with the vehicle shimmy system without clearance, the dynamic response of the vehicle shimmy system with clearance in universal joint is quite different. Therefore, it is necessary to take the clearance in the universal joint into consideration for modeling of the vehicle shimmy system, especially for those in-service vehicles with great clearance due to wear.

(2) Based on the presented numerical examples, it is found that the dynamic response of shimmy system with clearance experiences the variation from limit cycle, multi period motion, to chaos with increase of the clearance. The variation is dominantly originated from the variation of the transmission characteristic of the universal joint with clearance.

(3) The clearance in universal joint and the vehicle speed have coupling effects on the dynamic behavior of the vehicle shimmy system. The coupling effects of the parameters on the dynamic behavior of the system will be discussed in future research.

\section{Acknowledgments}

This research was supported by the Program for New Century Excellent Talents in University under grant NCET10-0358, the National Natural Science Foundation of China under grant 50975071, and the Changzhou Research Program of Application Foundation under grant CJ20110006. These supports are gratefully acknowledged.

\section{References}

[1] M. Demic, Analysis of influence of design parameters on steered wheels shimmy of heavy vehicle, Vehicle System Dynamic 26(5) (1996), 343-379.

[2] A.P. Kovacs, Computational vibration analysis of vehicle shimmy by a power-work method, Vehicle System Dynamics 29(6) (1998), 341-364.

[3] G. Stepan and B. Goodwine, Controlling unstable rolling phenomena, Journal of Vibration and Control 6(1) (2000), $137-158$.

[4] J.T. Gordon, Perturbation analysis of nonlinear wheel shimmy, Journal of Aircraft 39(2) (2002), 305-317.

[5] Y. Lin and S. Li, Study on the bifurcation character of steering wheel self-excited shimmy of motor vehicle with dependent suspension, Chinese Journal of Mechanical Engineering 40(12) (2004), 187-191.

[6] A. Virgile, C. Vinod and J. Nader, Experimental testing and validation of tangential steering wheel vibrations due to tire nonuniformity, ASME Dynamic Systems and Control Division 74(1) (2005), 545-551.

[7] J.X. Zhou and L. Zhang, Incremental harmonic balance method for predicting amplitudes of a multi-DOF, nonlinear wheel shimmy system with combined coulomb and quadratic damping, Journal of Sound and Vibration 279(1-2) (2005), 403-416.

[8] H.J. Che, N. Chen and G.D. Yin, Optimum design of wheel shimmy control for a kind of off - road vehicle based on genetic algorithm, Chinese Mechanical Engineering 17(24) (2006), 2635-2638, (in Chinese).

[9] A.E. Belkin, B.L. Bukhin, O.N. Mukhin et al., Some models and methods of pneumatic tire mechanics, Vehicle System Dynamics 27(Supp) (1997), 250-271.

[10] Z. Liu, G. Payre and P. Bourassa, Nonlinear oscillations and chaotic motions in a road vehicle system with driver steering control, Nonlinear Dynamics 9(3) (1996), 281-304.

[11] S. Li and Y. Lin, Numerical analysis on the bifurcation character of automotive steering wheel, Journal of Jilin University (Engineering Science) 35(1) (2005), 7-11, (in Chinese).

[12] J. Xin, Dynamic analysis of vehicle shimmy system with stochastic clearance in movement pair of steering linkage, Master dissertation, Hefei University of Technology, Hefei, China (in Chinese), 2011.

[13] S. Zhang, Dynamic analysis of vehicle shimmy system with clearance in steering handling mechanism, Master dissertation, Hefei University of Technology, Hefei, China (in Chinese), 2010.

[14] J. Lu, J. Gu and M. Liu, Modeling of the vehicle shimmy system with consideration of clearance of the steering mechanism, Meccanica 45(1) (2010), 53-61. 

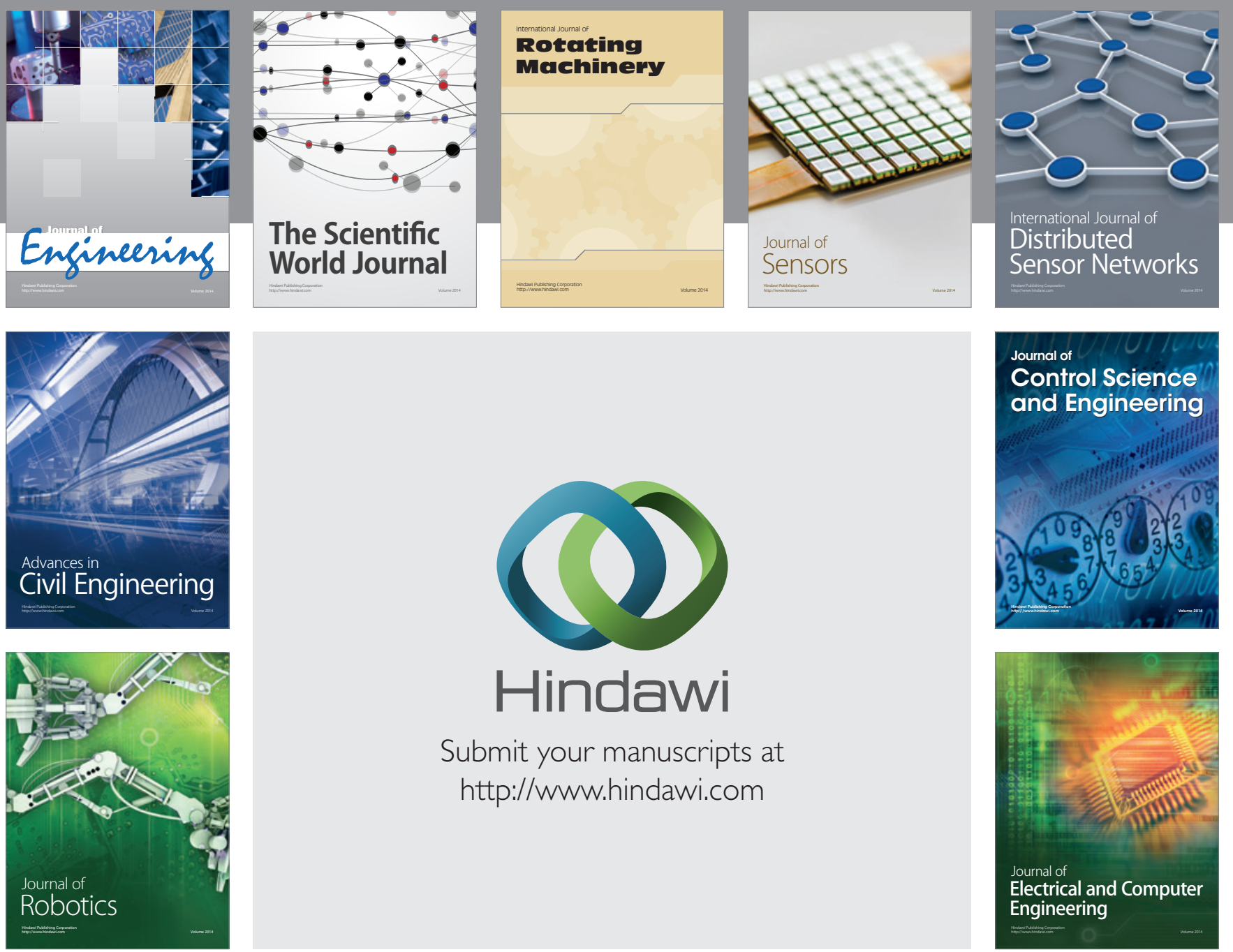

Submit your manuscripts at

http://www.hindawi.com
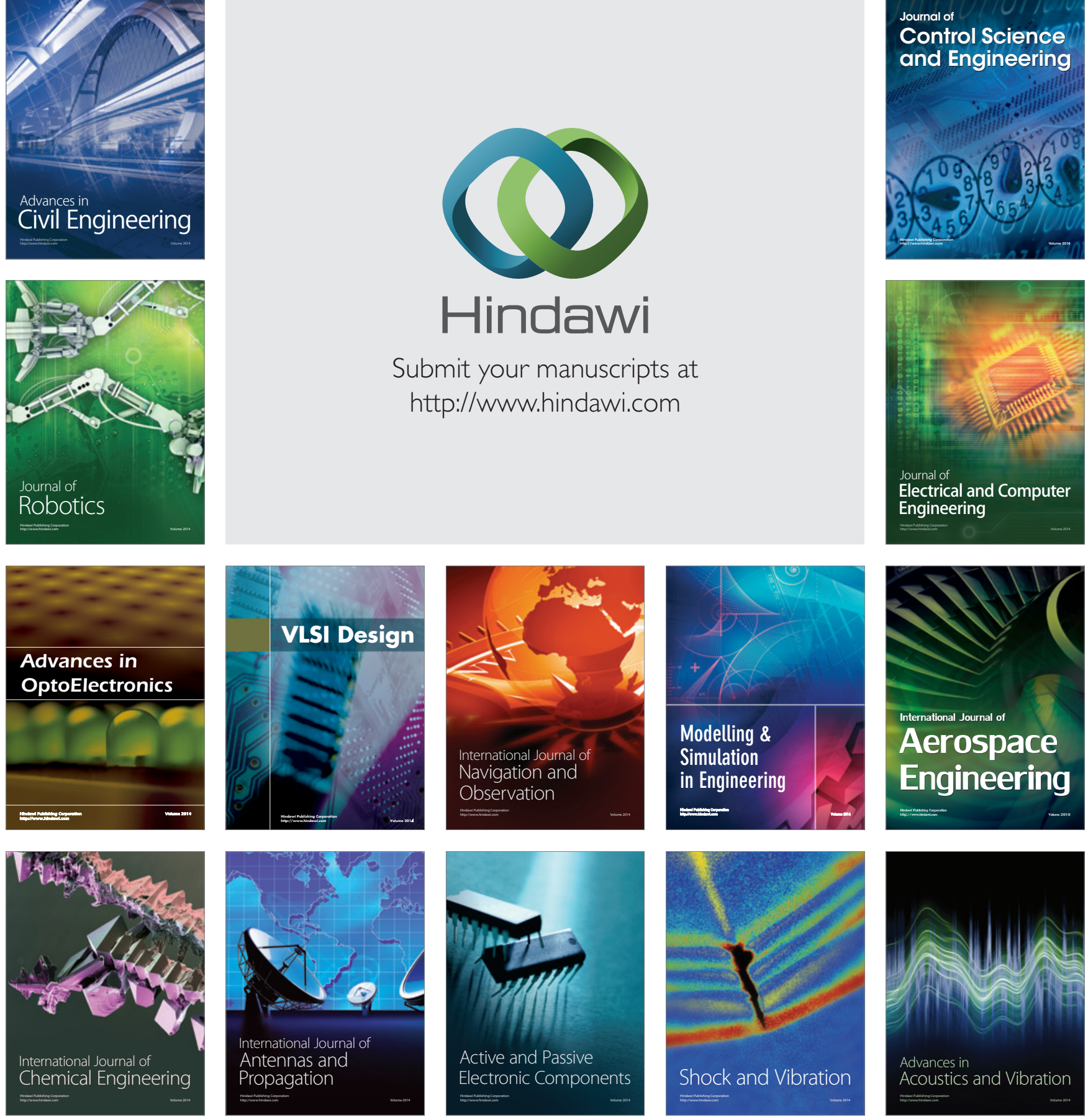